\title{
Infant hydrocephalus in sub-Saharan Africa: the reality on the Tanzanian side of the lake
}

\author{
Maria M. Santos, MD, ${ }^{1}$ Derick K. Rubagumya, ${ }^{4}$ Imani Dominic, MD, ${ }^{4}$ Amos Brighton, MD, ${ }^{4}$ \\ Soledad Colombe, DVM, MPH, ${ }^{2}$ Philip O'Donnell, BA, ${ }^{3}$ Micaella R. Zubkov, BS, ${ }^{1}$ and \\ Roger Härtl, MD'1
}

${ }^{1}$ Department of Neurological Surgery, Weill Cornell Brain and Spine Center, and ${ }^{2}$ Center for Global Health, ${ }^{3}$ Weill Cornell Medical College, NewYork-Presbyterian, New York, New York; and ${ }^{4}$ Catholic University for Health and Allied Sciences, Bugando Medical Centre, Mwanza, Tanzania

OBJECTIVE Infant hydrocephalus is estimated to affect more than 100,000 new infants each year in sub-Saharan Africa (SSA). Bugando Medical Centre (BMC), a government-funded and patient cost-shared referral center, serves over 13 million people in the Lake and Western regions of Tanzania. The goals of this study were to characterize the infant population affected by hydrocephalus who presented to BMC and were treated with a ventriculoperitoneal shunt (VPS) to determine the rate of early complications associated with this surgical procedure and to assess its potential risk factors.

METHODS Data were prospectively collected from all patients less than 1 year of age who, over a period of 7 months, were diagnosed with hydrocephalus and admitted to BMC for insertion of a primary VPS. Demographic data, maternal history, preoperative studies, surgical procedure, and surgical complications developing by the time of the first follow-up visit were analyzed. Risk factors associated with the surgical complications were determined.

RESULTS During the 7-month study period, 125 infants eligible for the study were included in the analysis. Overall, $75 \%$ were younger than 6 months of age, and $56 \%$ were males. Only $7 \%$ of mothers had a gestational ultrasound, $98 \%$ did not receive preconception folic acid, and $25 \%$ delivered their child at home. In most patients with hydrocephalus the etiology was uncertain (56\%), and other patients had postinfectious $(22.4 \%)$ or myelomeningocele-associated (16\%) hydrocephalus. Patients' mean head circumference on admission was $51.4 \pm 6.3 \mathrm{~cm}$. Their median age at shunt surgery was 137 days, and $22.4 \%$ of the patients were operated on without having undergone radiological assessment. The majority of shunts were placed in a right parietooccipital location. Thirteen patients had undergone a previous intraventricular endoscopic procedure. Overall, at least one surgical complication was found in $33.6 \%$ of patients up to the first follow-up assessment (median follow-up time of 70 days); shunt infection was the most common complication. The postoperative mortality rate was $9 \%$. The risk factors associated with early surgical complications were tumor-related etiology, larger head circumference, and postoperative hospital stays of greater duration.

CONCLUSIONS In a region of the continent where most infant hydrocephalus cases had an uncertain etiology, most patients presented to the hospital in a late stage, with no prenatal diagnosis and with large head circumferences. Standard preoperative investigations were not uniformly performed, and the surgical complications, led by VPS infection, were disturbingly high. Younger patient age, previous endoscopic procedure, surgeon involved, and cranial location of the VPS had no statistical relation to the surgical complications.

This study shows that the positive results previously reported by SSA mission hospitals, subspecialized in pediatric neurosurgery, are still not generalizable to every hospital in East Africa. To improve maternal and neonatal care in the Lake region of Tanzania, the development of a fluxogram to determine hydrocephalus etiology, a strict perioperative protocol for VPS insertion, and an increase in the number of endoscopic procedures are recommended to BMC.

https://thejns.org/doi/abs/10.3171/2017.5.PEDS1755

KEY WORDS infant hydrocephalus; sub-Saharan Africa; ventriculoperitoneal shunt; Tanzania

ABBREVIATIONS BKKH = Bethany Kids at Kijabe Hospital; $\mathrm{BMC}=$ Bugando Medical Centre; $\mathrm{CPC}=$ choroid plexus cauterization; $\mathrm{CSF}=$ cerebrospinal fluid; ETV = endoscopic third ventriculostomy; HIV = human immunodeficiency virus; IQR = interquartile range; MMC = myelomeningocele; SSA = sub-Saharan Africa; VPS = ventriculoperitoneal shunt.

SUBMITTED February 12, 2017. ACCEPTED May 15, 2017.

INCLUDE WHEN CITING Published online September 8, 2017; DOI: 10.3171/2017.5.PEDS1755. 
$\mathrm{I}$ N sub-Saharan Africa (SSA), infant hydrocephalus is estimated to affect more than 100,000 new infants each year. ${ }^{16}$ Tanzania, a sub-Saharan country, has a population of approximately 55 million inhabitants, and only 9 board-certified neurosurgeons. Seven of these neurosurgeons work in Dar es Salaam, one is employed in Mwanza, and one has emigrated to Botswana. Tanzania is considered the 25 th poorest country in the world; it has a gross domestic product based on purchasing-power-parity per capita of 2,054.32 USD and has one of the highest birth rates in the world (https://www.gfmag.com/globaldata/country-data/tanzania-gdp-country-report). ${ }^{13}$

Bugando Medical Center (BMC), located in Mwanza, is one of two tertiary-care hospitals in the country that provides neurosurgical care in the rural Lake and Western regions of Tanzania. The exact incidence of infant hydrocephalus in Tanzania is unknown, but BMC inserts approximately 400 ventriculoperitoneal shunts (VPS) per year, approximately $60 \%$ of which are primary insertions in infants. Before May 2016, BMC had no intraventricular endoscope, and the great majority of the infants presenting with hydrocephalus were treated with a VPS. The placement of a VPS in an infant has a complication rate as high as $40 \%$, even in the best hands and in the most accomplished health care centers. ${ }^{3}$ Moreover, the need for a VPS implies that the patient will require life-long follow-up care, which is compromised by the low accessibility of hospitals in rural Africa.

In SSA, the majority of the up-to-date reports regarding infant hydrocephalus and its treatment have been written by two pediatric neurosurgeons, Benjamin Warf and Leland Albright, who both worked at nonprofit referral centers for pediatric neurosurgery in Uganda (CURE Children's Hospital of Uganda, Mbale) and Kenya (Bethany Kids at Kijabe Hospital [BKKH], Kijabe), respectively. Both authors reported complication rates associated with VPS insertion similar to those seen in developed countries, with rates of VPS infection of $9.7 \%{ }^{15}$ and $9.1 \%$, respectively. ${ }^{5}$ The only Tanzanian study (Muhimbili University College of Health Sciences, Dar es Salaam) that reported complications related to VPSs in children was conducted almost 20 years ago, and it showed a combined wound and VPS infection rate of $46.1 \% .^{6}$ Based on these data and on data from our own intuition, we reasonably assume that VPS complication rates reported by ultra-specialized mission hospitals are not reproducible by the most health facilities in SSA, which constitute the majority of the health care providers in this subcontinent region.

The present study had two main goals: 1) to characterize in detail the population of infants affected by hydrocephalus in the Lake region of Tanzania who presented to a government-funded hospital and were treated with a VPS, and 2) to determine the early complication rate associated with VPS placement at BMC and assess the relevant risk factors.

It is our hope that comparing the data collected at BMC with data from previous reports will lead to an improvement of the pediatric neurosurgical care in the Lake region of Tanzania.

\section{Methods}

After receiving IRB approval, we prospectively col- lected data from all patients less than 1 year of age who were diagnosed with hydrocephalus and were admitted to BMC, Mwanza, Tanzania, for primary insertion of a VPS from February 2016 through August 2016. Data included patient demographics, region of birth, and interval between diagnosis and treatment, as well as findings from preoperative assessments (e.g., hydrocephalus etiology, head circumference, radiological findings, and serum hemoglobin level), operative detail (e.g., surgical protocol, duration of postoperative stay, morbidity, and mortality), and maternal history/neonatal history (e.g., attendance of antenatal clinic, preconception supplementation of folic acid, existence of gestational ultrasounds, mode of delivery, place of delivery, gestational age at delivery, history of neonatal sepsis, and maternal human immunodeficiency virus [HIV] serostatus). We excluded patients who were older than 1 year of age; who had undergone a shunt revision, external ventricular drainage, or cerebrospinal fluid (CSF) reservoir placement; or who had undergone an endoscopic procedure with no shunt placement. Patients who had undergone a previous surgery but still had a primary VPS insertion were included in the analysis. All data were recorded on Microsoft Excel sheets.

The etiology of hydrocephalus was divided as follows: uncertain (including likely congenital and undiagnosed postinfectious, according to the criteria used), postinfectious, secondary to myelomeningocele (MMC), secondary to a CNS tumor, and prematurity-associated hydrocephalus. The category "uncertain" was subdivided into the following: aqueductal stenosis, atresia of foramina of Monro, atresia of the exit foramina of the fourth ventricle, Dandy-Walker malformation, and encephalocele. The classification of "postinfectious" hydrocephalus was considered when there was a medical history of hospital (inpatient)-treated febrile illness and subsequent macrocephaly.

Surgical morbidity was assessed up to the first followup appointment. It was not the intention of this study to determine the rate of VPS insertion-related complications over time (calculated as $43.6 \%$ at 2 years post-shunt placement $^{3}$ ) but rather to assess the complication rate up to the first follow-up appointment, knowing a priori that this rate would be lower than the one that would be reported were a longer follow-up period to be considered.

\section{Statistical Analysis}

Statistical analysis was done using both Microsoft Excel and R. Categorical variables were described as proportions, and continuous variables were described using the mean \pm standard deviation or the median and interquartile range (IQR; quartiles 1-3), depending on the distribution of the data. Associations between the cause of hydrocephalus and potential risk factors were tested for by using Fisher's exact tests. Associations between the complications up to the first follow-up visit and potential risk factors were tested for by using univariate logistic regression and ANOVA. Significance was set at $\mathrm{p}<0.05$.

\section{Characterization of the Perioperative Environment}

BMC, a 900-bed tertiary-care hospital in Mwanza, 
Tanzania, was opened in 1971 and currently serves approximately 13 million people. Since its opening, it has been the referral center for the Lake and Western Zones of the United Republic of Tanzania-namely the regions of Mwanza, Simiyu, Mara, Kagera, Shinyanga, Tabora, and Kigoma. It is owned by the Episcopal Conference of Catholic Bishops of Tanzania, and it is directed in partnership with the national government. Funding comes exclusively from the government in a cost-sharing arrangement with patients.

The neurosurgery department of BMC was officially created in October 2015 and has a permanent staff of 1 Tanzanian neurosurgeon, 3 medical officers, and a rotating team of general interns and general surgery residents. The pediatric neurosurgery ward is integrated into an adult general surgical ward with no specialized neurosurgical nursing care.

Preoperatively, most patients undergo a head ultrasound (transfontanel) and serum hemoglobin assessment; CSF is rarely analyzed. Patients younger than 5 years of age are not charged for hospital costs, except for radiological investigations and medications.

Intraoperatively, anesthesia is mostly administered by nurses, given that there are only 2 licensed local anesthesiologists in the hospital; halothane is used in the majority of cases. The VPS that is available is the Chhabra medium-pressure Slit N Spring valve (Surgiwear), donated by the International Federation for Spina Bifida and Hydrocephalus. These valves are placed by default mostly in the right parietooccipital region. Whether intravenous antibiotic agents are administered is dependent on availability of the drugs. The supply of vancomycin is limited at the hospital's pharmacy and is sold at a high price at community pharmacies. The operating room where these infants undergo surgery is a common space shared by several specialties and has no restrictions on how people circulate throughout the area. Surgeons wear double gloves; the gowns and drapes are made of cloth.

\section{Results}

The analysis included 125 infants who were newly diagnosed with hydrocephalus, presented to BMC, and received a VPS between February 1 and August 31, 2016. These infants were followed up over a median of 70 days (IQR 49-126 days), with a minimum follow-up of 21 days and a maximum of 266 days. Males composed $56 \%$ of the population. Ages ranged between 4 and 365 days with a median age of 106 days (IQR 61-174.2 days) upon admission; $75 \%$ of patients were younger than 6 months. Over half of the patients came from the regions of Mwanza (26.4\%), Tabora (15.2\%), and Shinyanga (14.4\%); patients were also referred at smaller numbers from 7 other regions. Neither sex nor age was associated with the incidence of postoperative complications up to the first follow-up ( $\mathrm{p}=$ 0.77 and $\mathrm{p}=0.41$, respectively).

Regarding the gestational history, $98.3 \%$ of mothers reported having attended the antenatal clinic in their home region at least once, $97.5 \%$ of mothers did not receive folic acid before conception, and only $6.8 \%$ received at least one prenatal ultrasound. Vaginal delivery $(89.9 \%)$ was
TABLE 1. Summary of hydrocephalus etiologies in 125 patients

\begin{tabular}{lc}
\hline \multicolumn{1}{c}{ Etiology of Hydrocephalus } & No. of Patients (\%) \\
\hline $\begin{array}{l}\text { Uncertain (presumed congenital \& undiag- } \\
\text { nosed postinfectious) }\end{array}$ & $70(56)$ \\
\hline Dandy-Walker spectrum & 4 \\
\hline Atresia of foramina of Monro & 7 \\
\hline Atresia of the exit foramina of the 4th ventricle & 2 \\
\hline Aqueductal stenosis & 4 \\
\hline Encephalocele & 1 \\
\hline Holoprosencephaly & 1 \\
\hline Syndrome associated & 1 \\
\hline Unknown & 50 \\
\hline Postinfectious & $28(22.4)$ \\
\hline Myelomeningocele & $20(16)$ \\
\hline Tumor & $3(2.4)$ \\
\hline Unclassifiable & $4(3.2)$ \\
\hline
\end{tabular}

more common than caesarean section; $25.4 \%$ of births occurred at home without any specialized assistance. Nevertheless, home delivery was not associated with postinfectious hydrocephalus. The vast majority of infants were full term $(90.7 \%)$. A positive history of infant sepsis motivated $23.2 \%$ of mothers to seek hospital admission and a course of intravenous antibiotics. HIV serostatus was assessed in $79.2 \%$ of mothers, $4 \%$ of whom were positive. Maternal HIV status was not significantly associated with the presence of postoperative complications $(\mathrm{p}=0.56)$.

The etiologies of hydrocephalus were characterized as follows: uncertain (including presumed congenital and undiagnosed postinfectious hydrocephalus), which accounted for the majority of the cases $(56 \%)$, followed by the postinfectious type (22.4\%), MMC-associated (16\%), and tumor-related hydrocephalus (2.4\%) (Table 1). In 3.2\% of the cases, the mothers did not provide a clear history, and the etiology was then classified as "non-classifiable." There was no overall significant association between hydrocephalus etiology and type of complication $(p=0.58)$. However, there was an overall association between hydrocephalus etiology (tumor-related type) and postoperative complications $(\mathrm{p}=0.0121)$.

Head circumference was assessed and plotted in $74.4 \%$ of patients. The mean head circumference was $51.4 \pm 6.3$ $\mathrm{cm}$. Preoperative head circumference was significantly associated with surgical complications $(\mathrm{OR}=0.90$ [95\% CI $0.82-0.98], \mathrm{p}=0.021)$. Overall 95 patients $(76 \%)$ underwent preoperative ultrasound; 2 patients $(1.6 \%)$ underwent preoperative CT scanning; and the remaining 28 patients (22.4\%) did not receive any radiological assessment. Preoperative serum hemoglobin concentration was assessed in $67.2 \%$ of patients. Serum hemoglobin ranged between 4.3 and $18.7 \mathrm{~g} / \mathrm{dl}$, with a median of $9.75 \mathrm{~g} / \mathrm{dl}$ (IQR 8.5-11.8 $\mathrm{g} / \mathrm{dl})$. Preoperative hemoglobin level was not associated with postoperative complications $(\mathrm{p}=0.996)$. In 4 patients a ventricular tap was performed either to collect CSF samples or to relieve the intracranial pressure and temporize a CSF diversion.

The median age at shunt surgery was 137 days (ap- 
TABLE 2. Type of surgeries performed previous to VPS implantation

\begin{tabular}{lc}
\hline \multicolumn{1}{c}{ Previous Surgery } & No. of Patients $(\%)^{*}$ \\
\hline Endoscopic procedure & $13(52)$ \\
\hline Abscess drainage & $2(8)$ \\
\hline MMC repair & $7(28)$ \\
\hline Encephalocele repair & $1(4)$ \\
\hline ETV & $2(8)$ \\
\hline
\end{tabular}

* Previous surgeries were performed in 25 patients.

proximately 4 months; IQR 90-200 days). The youngest patient was 20 days old. The median time in between neurosurgical referral and surgery was 15 days (IQR 8-38 days). The delay in the surgical procedure was not associated with postoperative complications $(\mathrm{p}=0.22)$. Regarding the anatomical location, $88 \%$ of shunts were right parietooccipital, 9.6\% were right frontal, $1.6 \%$ were left parietal, and $0.8 \%$ were left frontal. The cranial entry point was not significantly associated with postoperative complications $(\mathrm{p}=0.07)$, but there appeared to be a trend of increased complication with frontal locations (mainly hardware exposure).

For most patients (80\%), VPS placement was their first surgery. Nevertheless, $20 \%$ had undergone a previous surgery, of which $52 \%$ were endoscopic procedures and $28 \%$ were MMC repairs (Table 2). A history of surgery was not associated with complications $(\mathrm{p}=0.57)$. There were 21 different combinations of the surgical team. At least one neurosurgeon participated in $51.2 \%$ of the operations. Medical officers and general surgery residents performed the remaining operations. The composition of the surgical team was also not associated with postoperative complications $(\mathrm{p}=0.07)$.

The median duration of postoperative stay was 4 days (IQR 3-6 days). Longer postoperative stays were positively associated with complications up to first follow-up $(\mathrm{OR}=1.19$ [95\% CI 1.05-1.39]; $\mathrm{p}=0.016)$. There was at least one follow-up assessment in $88 \%$ of the patients. The mean time to first postoperative visit was $36.9 \pm 19.4$ days.

Overall, at least one surgical complication was found in $33.6 \%(n=37)$ of patients up to the first follow-up assessment. A surgical complication (morbidity) was defined as any complication that occurred intraoperatively or during the postoperative period that related to the surgical procedure itself or to the condition of the patient and was the motivation for the surgical procedure.

Intraoperative surgical complications occurred in $2.4 \%$ of patients $(n=3)$ : a prolonged cardiac arrest in 1 patient, a rapid outflow of CSF, which caused dural detachment and prohibited ventricular catheterization in a second patient, and a tension pneumothorax in a third patient.

Early postoperative complications were found in $1.6 \%$ of patients $(n=2)$. Both patients died of presumptive respiratory failure in the first 24 hours after surgery.

Other postoperative complications at first follow-up were categorized as infectious, mechanical, and wound problems (Table 3). VPS infection was the most common
TABLE 3. Surgery-related complications diagnosed at first follow-up

\begin{tabular}{cc}
\hline Surgical Complication & No. of Patients (\%) \\
\hline Infection & $15(13.6)$ \\
\hline Hardware exposure & 7 \\
\hline Shunt malfunction, fever & 5 \\
\hline Pus infiltration of shunt tract & 3 \\
\hline Mechanical & $10(9)$ \\
\hline Proximal shunt malfunction & 8 \\
\hline Shunt migration & 1 \\
\hline Anal protrusion of the shunt & 1 \\
\hline Wound problems & $7(6.4)$ \\
\hline Superficial scalp infection & 3 \\
\hline Abdominal wound infection & 1 \\
\hline Scalp \& abdominal wound infection & 1 \\
\hline CSF leak & 1 \\
\hline Dehiscence of abdominal wound & 1 \\
\hline * Follow-up data were available for 110 (88\%) of the 125 patients.
\end{tabular}

* Follow-up data were available for 110 (88\%) of the 125 patients.

complication (15 [13.6\%] of 110 patients), followed by mechanical complications (10 [9\%]) and wound problems (7 [6.4\%]). Most patients with shunt infections presented with hardware exposure after the shunt ulcerated through the skin (7 [46.7\%] of 15 patients), shunt malfunction with systemic signs of infection (5 [33.3\%]), and shunt tract infiltration with pus (3 [20\%]). Most mechanical complications were proximal shunt malfunctions (8 [80\%] of 10 patients), but 1 patient (10\%) presented with VPS dislocation, and another patient (10\%) presented with anal protrusion of the shunt. The most common wound problems were superficial scalp wound infection $(n=3)$, but 1 patient presented with an abdominal wound infection and another presented with both scalp and abdominal wound infections. Finally, there was 1 CSF leak and 1 dehiscence of the abdominal suture that required intervention.

The overall mortality rate was $14.5 \%$. The postoperative mortality rate (defined as death before postoperative day 30 or recognizably related to the surgical intervention) was $9 \%$. Three deaths were not correlated with the surgical procedure or the basal condition of the patient; in another 3 cases, the cause of death was indeterminable (Table 4).

\section{Discussion}

Infant hydrocephalus represents a major burden of neurosurgical disease in SSA. ${ }^{16}$ VPS insertion remains the gold standard for hydrocephalus treatment at most hospitals in this subcontinental region, where the workforce is numerically limited ${ }^{4}$ and the infrastructure is poor and the patient load is enormous. Our study included a carefully chosen population that represents the majority of hydrocephalus cases-that is, patients younger than 1 year of age who presented to a government-funded referral hospital (the only one in the Lake and West regions of Tanzania), were newly diagnosed with hydrocephalus, and 
TABLE 4. Summary of cases in which patients died

\begin{tabular}{|c|c|c|c|c|}
\hline Patient No. & Cause of Death & Postop Day of Death & Surgical Complications & Cause of Hydrocephalus \\
\hline \multicolumn{5}{|l|}{ Died postop $(n=10)$} \\
\hline 1 & Respiratory failure & 1 & No & Unknown \\
\hline 2 & Sepsis & 72 & VPS infection & MMC \\
\hline 3 & VPS malfunction & 48 & Mechanical & Postinfectious \\
\hline 4 & Sepsis & 7 & VPS infection & Presumed congenital \\
\hline 5 & Sepsis & 161 & VPS infection & Presumed congenital \\
\hline 6 & Sepsis & 44 & VPS infection & Presumed congenital \\
\hline 7 & Aspiration pneumonia (Chiari II) & 138 & VPS infection & MMC \\
\hline 8 & Aspiration pneumonia & 60 & VPS infection & Presumed congenital \\
\hline 9 & Respiratory failure (Chiari II) & 1 & No & MMC \\
\hline 10 & Sepsis & 15 & VPS infection & Postinfectious \\
\hline \multicolumn{5}{|c|}{ Death unrelated to op $(n=3)$} \\
\hline 1 & Malaria & 50 & No & MMC \\
\hline 2 & Malaria & 52 & No & Presumed congenital \\
\hline 3 & Malaria & 22 & No & MMC \\
\hline \multicolumn{5}{|c|}{ Cause of death unknown $(n=3)$} \\
\hline 1 & Died at home & 61 & VPS infection & MMC \\
\hline 2 & Died at home & 35 & Wound & Presumed congenital \\
\hline 3 & Died at home & 31 & No & Presumed congenital \\
\hline
\end{tabular}

were treated with primary VPS insertion. These narrow inclusion criteria homogenized the study population and provided information on a different patient group from those described in the majority of the previous studies, which included broader age ranges and both primary and revision VPS insertions.

\section{Demographics: Sex and Age}

Previously published African studies have already shown that the majority of pediatric patients with hydrocephalus were male-64.6\% of the population in a study from Tanzania, ${ }^{6} 60.5 \%$ in a study from Nigeria, ${ }^{7} 51.9 \%$ in a study from Uganda, ${ }^{8}$ and $53 \%$ in a study from Kenya. ${ }^{6}$ The findings in the present study are concordant, despite the lack of association between sex and VPS complications as previously reported by Gathura et al., who reported that females were more prone to surgical complications, ${ }^{5}$ and Mwangombe ${ }^{11}$ et al., who reported the ratio of VPS infection in males versus females to be 1.3:1. These differing results may be attributed to the lack of power in the present study.

The association between younger age and surgical complications has also been reported, ${ }^{2,8,14}$ with patient age younger than 1 year being associated with higher rates of complications than patient age older than 1 year. For example, a study from Kenyatta National Hospital, which included patients from 3 months to 12 years of age, found that $50.6 \%$ of VPS infections occurred in patients younger than 1 year of age. ${ }^{11}$ In the present study, we found no association between younger age and a higher rate of surgical complications. This may be because all patients included were younger than 1 year of age, and the age threshold for decreased surgical complications may not be within the first 12 months of life. Gathura et al..$^{5}$ reported the same finding in a cohort of patients younger than 1 year of age.

\section{Maternal History}

Maternal histories were not analyzed in previous studies. The majority of maternal histories in the present study indicated unplanned conceptions, lack of birth control, lack of folic acid to supplement daily diets, and no access to gestational ultrasounds and thus no prenatal diagnosis. The lack of prenatal information did not allow us to confirm the "congenital" etiology as a cause for hydrocephalus, and so this category that could be overlooked was termed "uncertain." On the other hand, the poor perinatal care might have contributed to the high incidence of neonatal sepsis, which is an enormous public health problem in SSA with a cumulative incidence at birth of $6 \%$ and a case fatality rate of around $14 \% .^{12}$

\section{Hydrocephalus Etiology}

The predominance of "uncertain" etiology in the present study differs from the Ugandan ${ }^{17}$ and Kijabe ${ }^{5}$ studies, which reported postinfectious (60\%) and MMC-associated (43.4\%) types to be the most common etiologies for hydrocephalus, respectively. It also differs from the experiences from Zambia, Zimbabwe, Malawi, and Nairobi that identified congenital hydrocephalus to be the most common. ${ }^{1,11}$

The cases designated as "uncertain" in the present study did not fit the criteria to be considered "clearly" postinfectious; they could not be considered to be "congenital" given the absence of a prenatal diagnosis and postnatal imaging; and they were not associated with an MMC, a tumor, or an intraventricular hemorrhage. This category likely rep- 
resents a mixed etiological group comprising congenital cases (such as those presumed to be associated to a Dandy-Walker syndrome) and undocumented postinfectious cases; the distribution between them is, however, unknown.

In CURE Children's Hospital of Uganda, where the postinfectious etiology predominated, suggestive imaging and ventriculoscopic findings were taken into account when classifying the cause of hydrocephalus, as were a history of febrile illness or epilepsy and a negative history of hydrocephalus at birth. ${ }^{17}$ We found it extremely difficult to use the same criteria in the present study, given that only $1.6 \%$ of the population had undergone preoperative brain CT scanning and $22.4 \%$ did not undergo preoperative imaging, which precluded the use of findings from imaging. Also, only $10 \%$ of patients had a pre-VPS ventriculoscopy, which likewise precluded the use of ventriculoscopic findings. Finally, the neonatal histories provided by the mothers were usually poor (unplanned conceptions, lack of birth control, lack of folic acid, and no access to gestational ultrasounds and thus no prenatal diagnosis), and $25 \%$ of the infants were born at home and did not have any neonatal register. Therefore, the criteria used to define evident postinfectious hydrocephalus were that the patient had previously been admitted to a health care facility, that the patient had been treated with a course of intravenous antibiotics, and that this incident correlated with the timeline of increasing head circumference. Patients with a medical history of untreated febrile illness were not included in this group because it was considered unlikely that those affected with neonatal sepsis or meningitis had not ever been treated with intravenous antibiotics in the past. This approach obviously narrowed the classification of postinfectious hydrocephalus and likely indicates that postinfectious cases were in fact part of the "uncertain" etiological group.

At Bethany Kids at Kijabe Hospital (BKKH) in Kenya, the most common hydrocephalus etiology was MMC related. The results of a study by authors from BKKH were primarily biased by the fact that this hospital is a major referral center for patients with MMC; on the other hand, the mean head circumference for shunt-treated patients was $46.5 \pm 8.4 \mathrm{~cm}$ (90th percentile). ${ }^{5}$ At $\mathrm{BMC}$, we had tended not to insert a shunt in patients with MMC and macrocephaly unless they had exhibited consistent signs of intracranial hypertension separate from an isolated macrocephaly, clinical signs of Chiari Type II malformation, or head circumference crossing percentiles over time.

Unclassifiable cases of hydrocephalus were missing a maternal and neonatal history, which precluded any etiological classification.

Overall, hydrocephalus type in the present study was associated with the incidence of complications $(p=0.0121)$. Tumor-related hydrocephalus seemed to lead to more complications (100\% complication rate), although only 3 patients belonged to this category, which resulted in low power for the association. To the best of our knowledge, a relationship between tumor-associated hydrocephalus and complication rate had not yet been reported.

The present study did not show an association between a specific type of complication and a specific etiology. This result was unlike previous reports by Gathura et al., who found a higher association between MMC and mechanical complications, ${ }^{5}$ and Drake et al., who found a higher association between MMC and infectious complications. ${ }^{3}$ Once again, the low power of the current study does not allow for further conclusions.

\section{Head Circumference}

In the current study, head circumference was positively associated with surgical complications up to the first follow-up. We observed larger head circumference measurements in the present study than those reported by Gathura et al. ${ }^{5}$ and similar values to those reported by the group from Muhimbili Orthopedic Institute at Dar es Salaam ${ }^{6}$ and by the group from Uganda. ${ }^{14}$ If one assumes that head circumference is a measure of the severity of the hydrocephalus or at least a measure of the delay in seeking neurosurgical care, it can be inferred that patients treated at $\mathrm{BMC}$ were more severely afflicted and referred in a more delayed fashion. A large head circumference and a thinner scalp thickness are also often associated with malnutrition.

\section{Preoperative Radiological Studies}

Previous studies have focused on communities where radiological studies were free of charge. Gathura et al. reported that every patient who presented to BKKH with a suspected diagnosis of hydrocephalus underwent preoperative cranial ultrasonography and that $3 \%$ underwent brain CT scanning..$^{5}$ Additionally, according to the CUREUganda report, patients could obtain brain CT scans at Kampala at no cost. ${ }^{17}$

Radiological studies at BMC are not free of charge, which helps to explain the fact that nearly a quarter of patients in the present study had undergone shunt insertion without any preoperative imaging. At BMC, a brain ultrasound and a brain CT cost 25,000 Tanzanian shillings (approximately 11 USD) and 250,000 Tanzanian shillings (110 USD), respectively. Thus, the administration of imaging tests depended on the family's capacity to afford them.

Shunting an infant without preoperative imaging studies forced the assumption that every neonatal divergent macrocephaly was primarily caused by hydrocephalus, minimizing the importance of establishing the origin of the problem and its characterization and, consequently, foregoing personalization of the treatment.

\section{CSF Analysis and Hemoglobin}

Protocols for VPS insertion at Kijabe and Mbale were characterized by strict pre- and intraoperative criteria: $\mathrm{Pa}-$ tients should be afebrile and acceptably nourished, CSF white blood cell count should be less than 10 cells/ml, and hemoglobin level should be higher than $9 \mathrm{~g} / \mathrm{dl} . .^{5}$ Moreover, CSF was collected intraoperatively and further cultured for every patient undergoing VPS insertion. ${ }^{14}$ In contrast to this protocol, the present study did not consider CSF analysis and hemoglobin serum level as part of the selection criteria for VPS insertion. Preoperative or intraoperative CSF sampling was not routinely performed at BMC, largely due to logistical constraints, such as a stock shortage of CSF tubes, unavailability of CSF tubes in the op- 
erating room, loss of CSF samples, inefficient release of results, CSF exposure to high temperatures, and so on. It is possible, in this scenario, that a fraction of these infants had an unrecognized CSF infection at the time of the surgical procedure.

\section{Interval Between Diagnosis and Surgery}

In the Kenyatta study, patients experienced a considerable "delay" between diagnosis and surgery, with only $43.5 \%$ of patients receiving treatment within 3 months of diagnosis. The authors of this study hypothesized that the delay in treatment was one of the determining factors for shunt infection. ${ }^{11}$ Contrary to the Kenyatta study, the interval in the present study between neurosurgical referral and surgery was not significant and furthermore was not associated with postoperative complications up to the first follow-up.

\section{Surgery and Surgeons}

In the present study, the choice of the anatomical location for VPS insertion was determined by the surgeon's personal experience and not based on the patient's characteristics. Despite the fact that the incidence of hardware exposure was higher when placed in a frontal location as opposed to a parietal location, this difference was not statistically significant in the present study, and the anatomical location of the shunt was not associated with postoperative complications up to first follow-up $(\mathrm{p}=0.10)$, a finding that is in agreement with results published by Warf. ${ }^{14}$

It is our opinion that patients who presented with a larger head circumference might have benefitted from the shunt having been placed in a parietal location rather than in a frontal location, because this would have decreased the length of the shunt segment positioned directly beneath the patient's thin scalp.

In addition, in the present series, the surgical team itself involved in the VPS insertion was not a factor that was significantly associated with the surgical complication rate, a finding that had previously been reported in separate studies. ${ }^{5}$ Thus, the surgeon's skills or training did not seem to play as important a role in shunt survival as factors related to the hospital environment and the patients' health status in SSA.

\section{Previous Surgery}

Similar to the results reported by Warf, ${ }^{14}$ in whose study patients who underwent primary VPS insertion were compared with patients who had undergone a previous endoscopic surgery (whether ventriculoscopy or endoscopic third ventriculostomy [ETV] attempt) before the VPS insertion, we found that a previous endoscopic procedure was not associated with a higher complication rate up to first follow-up. This finding raises the question of whether a ventriculoscopy should precede a VPS placement for all patients. Despite the fact that it is known that both ETV and VPS are associated with high failure rates in infants, in a setting that has poor health care access, deficient nutritional status, and high rates of VPS infection, the avoidance of a life-long implant seems to confer a long-term survival advantage. A study of 550 African children demonstrated that the adding of choroid plexus cauterization (CPC) to the classic ETV could also significantly improve outcome compared with ETV alone for infants younger than 1 year of age. In this series, for all etiologies of hydrocephalus and all ages of children, ETV/CPC was successful in 174 of 255 (68\%), thus successfully avoiding shunt dependence in the majority of participants. ${ }^{15}$

It is not our intention in this paper to compare the success rates of VPS placement and endoscopic procedures but rather to consider other treatment options for hydrocephalus that can reduce the absolute number of VPS insertions and consequently their inherent complications.

\section{Surgical Complications}

The $33.6 \%$ overall complication rate in the current study at a mean follow-up of 36.9 days is nearly as high as the $34.5 \%$ complication rate reported by Warf ${ }^{14}$ at 1 -year follow-up. In the Warf study, the mean time between surgery and shunt infection ranged from 1.88 to 2.4 months. ${ }^{14}$ In the Kijabe study, the mean time from shunt insertion to first complication was $3.5 \pm 4.1$ months (range 0.1-18 months), and the complication rate continued to increase over the 2-year follow-up period. ${ }^{5}$ Knowing a priori that the rate of VPS complications increases with time, the rate of VPS shunt complications reported herein during a short-term follow-up period was extremely high and requires further analysis.

In the present study the intraoperative anesthesia-related complications could likely have been prevented in a setting with a more advanced infrastructure and fully trained staff. Studies have shown that significant perioperative morbidity and mortality in these settings is preventable and that the safe administration of anesthesia is severely limited. ${ }^{10}$ Currently, there are 26 licensed local anesthesiologists in Tanzania, bringing the anesthesiologist/population ratio to $1: 21,000,000$. Of these 26 Tanzanian anesthesiologists, 3 have been "brain drained" to India and Swaziland, 2 were exclusively dedicated to private practice, 5 became involved in exclusively administrative roles, and only 2 currently work at BMC.

The 2 patients who died in the early postoperative period both had uneventful surgical procedures. Perioperative nursing care is one of the most challenging issues in SSA. At BMC, contrary to ultra-specialized pediatric mission hospitals, nurses had no specific training in postoperative neurosurgical management; the patient/nurse ratio was elevated and the medical equipment for postoperative monitoring was only available in the intensive care unit.

Contrary to reports by Gathura et al. ${ }^{5}$ and Warf ${ }^{14}$ which cited mechanical complications to be the most common, the present study found that shunt infection was the most common complication up to first follow-up. In fact, in the present study, the short-term (mean 36.9 days) shunt infection rate $(13.6 \%)$ was higher than the rates reported by Gathura et al. and Warf, and even follow-up periods were longer (mean 9 months ${ }^{5}$ with a $9.1 \%$ infection rate and a mean of 12 months with a $9.7 \%$ infection rate, ${ }^{14}$ respectively).

However, the current study results are similar to those of other SSA centers that are not ultra-specialized: in a study 
from Kenyatta (1982-1991), the infection rate was $24.6 \%$ over a 10 -year period; ${ }^{11}$ a Nigerian study reported an infection rate of $19.4 \%$ and a total complication rate of $25.8 \% ;{ }^{9}$ and Kinasha et al. reported a shunt infection rate of $24.6 \%$ and a combined shunt and wound infection rate of $40.6 \%{ }^{6}$

\section{Follow-Up}

Follow-up is a major issue in SSA, where geographic, economic, and logistic constraints limit access to health care centers. The $88 \%$ follow-up rate in the present study is likely a reflection of the short period of time. On the other hand, ultra-specialized pediatric neurosurgery centers that employ social workers who can visit patients at home report higher follow-up rates than local hospitals: $76 \%$ with a mean follow-up period of $8.9 \pm 6.6$ months in one study ${ }^{7}$ and $90.3 \%$ in a period 12 months in another, ${ }^{14}$ as compared with local hospitals whose mean follow-up rate was reported as $63.7 \%$ (mean follow-up period not specified). ${ }^{6}$

\section{Mortality}

The overall mortality rate in the present study (14.5\%) was higher than the rate reported by Gathura el al. $(7.1 \%)^{5}$ despite our shorter study period, and it was similar to the mortality rates reported by Warf ${ }^{14}(15.9 \%$ in up to 12 months of follow-up) and by Kinasha et al. ${ }^{6}$ (20\%, followup duration not specified). The $9 \%$ postoperative mortality rate in the present study was, in most cases, associated with previous surgical complications and particularly with VPS infections. The high mortality rate may be a reflection of the same factors that justify the high complication rate, such as poor nutritional status, lack of parental education and sense of awareness, poor hygiene, and poor access to health facilities.

\section{Conclusions}

At BMC, where the practice is cost-shared by patients and the government and the referral area is one populated with more than 13 million people, most infants admitted had hydrocephalus that was the result of unplanned and unsupervised gestations. Given the lack of prenatal diagnostic techniques and absence of postnatal imaging and ventriculoscopic findings, the cause of most hydrocephalus cases was uncertain. Nevertheless, the overall analysis indicates that infants presenting to the hospital late after hydrocephalus onset and with large head circumferences were significantly more likely to experience early surgical complications. Regarding the preoperative assessments, CSF analysis was rarely performed and not every patient underwent diagnostic radiological examination. Despite the caseload, the waiting period for surgery was not long, and neither the surgical team nor a previous endoscopic procedure impacted the shunt survival. The complication rate up to first follow-up was disturbingly high, with VPS infections being the leading complication and the most determinant risk factor for death.

In summary, the high rates of VPS complications seemed to be the product of a nonorganized system, a nonsystematic approach to characterization of the hydroceph- alus etiology, lack of perioperative protocols, and absence of perioperative nursing and anesthesiology training. To invert these results, it is important to develop maternal and neonatal care in the Lake region of Tanzania, to implement a strict perioperative protocol for shunt insertion at $\mathrm{BMC}$, and to invest in training of specialized nursing and anesthesia providers. The fact that a previous endoscopic procedure did not influence shunt survival expands the role of endoscopy, opens a whole new world of hydrocephalus treatment options, and puts into question the direct approach of inserting a VPS without attempting an initial endoscopic procedure. Hydrocephalus care providers should also be trained in intraventricular endoscopy concurrently with the implementation of VPS protocols.

\section{References}

1. Adeloye A: Management of infantile hydrocephalus in Central Africa. Trop Doct 31:67-70, 2001

2. Di Rocco C, Massimi L, Tamburrini G: Shunts vs endoscopic third ventriculostomy in infants: are there different types and/or rates of complications? A review. Childs Nerv Syst 22:1573-1589, 2006

3. Drake JM, Kestle JR, Milner R, Cinalli G, Boop F, Piatt J Jr, et al: Randomized trial of cerebrospinal fluid shunt valve design in pediatric hydrocephalus. Neurosurgery 43:294-305, 1998

4. El Khamlichi A: Neurosurgery in Africa. Clin Neurosurg 52:214-217, 2005

5. Gathura E, Poenaru D, Bransford R, Albright AL: Outcomes of ventriculoperitoneal shunt insertion in Sub-Saharan Africa. J Neurosurg Pediatr 6:329-335, 2010

6. Kinasha ADA, Kahamba JF, Semali IT: Complications of ventriculoperitoneal shunts in children in Dar es Salaam. East Cent Afr J Surg 10:55-59, 2005

7. Komolafe EO, Adeolu AA, Komolafe MA: Treatment of cerebrospinal fluid shunting complications in a Nigerian neurosurgery programme. Case illustrations and review. Pediatr Neurosurg 44:36-42, 2008

8. Lane JD, Mugamba J, Ssenyonga P, Warf BC: Effectiveness of the Bactiseal Universal Shunt for reducing shunt infection in a sub-Saharan African context: a retrospective cohort study in 160 Ugandan children. J Neurosurg Pediatr 13:140-144, 2014

9. Laurence FL: Treatment of hydrocephalus. East Cent Afr J Surg 11:78-80, 2006

10. Linden AF, Sekidde FS, Galukande M, Knowlton LM, Chackungal S, McQueen KA: Challenges of surgery in developing countries: a survey of surgical and anesthesia capacity in Uganda's public hospitals. World J Surg 36:1056-1065, 2012

11. Mwang'ombe NJM, Omulo T: Ventriculoperitoneal shunt surgery and shunt infections in children with non-tumor hydrocephalus at the Kenyatta National Hospital, Nairobi. East Afr Med J 77:389-390, 2000

12. Seale AC, Blencowe H, Manu AA, Nair H, Bahl R, Qazi SA, et al: Estimates of possible severe bacterial infection in neonates in sub-Saharan Africa, south Asia, and Latin America for 2012: a systematic review and meta-analysis. Lancet Infect Dis 14:731-741, 2014

13. United Republic of Tanzania: 2012 Population and Housing Census. Dar es Salaam, Tanzania: National Bureau of Statistics, 2014

14. Warf BC: Comparison of 1-year outcomes for the Chhabra and Codman-Hakim Micro Precision shunt systems in Uganda: a prospective study in 195 children. J Neurosurg 102 (4 Suppl):358-362, 2005 
15. Warf BC: Comparison of endoscopic third ventriculostomy alone and combined with choroid plexus cauterization in infants younger than 1 year of age: a prospective study in 550 African children. J Neurosurg 103 (6 Suppl):475-481, 2005

16. Warf BC: Educate one to save a few. Educate a few to save many. World Neurosurg 79 (2 Suppl):S15.e15-S15.e18, 2013

17. Warf BC: Hydrocephalus in Uganda: the predominance of infectious origin and primary management with endoscopic third ventriculostomy. J Neurosurg 102 (1 Suppl):1-15, 2005

\section{Disclosures}

Dr. Härtl is a consultant for DuPuy-Synthes, Brainlab, and Ulrich.

\section{Author Contributions}

Conception and design: Santos. Acquisition of data: Santos, Rubagumya, Dominic, Brighton, O’Donnell. Analysis and interpretation of data: Santos, Colombe. Drafting the article: Santos. Critically revising the article: Härtl, Rubagumya, Dominic, Brighton, Colombe, O'Donnell, Zubkov. Reviewed submitted version of manuscript: Härtl, Colombe, Zubkov. Approved the final version of the manuscript on behalf of all authors: Härtl. Statistical analysis: Colombe. Administrative/technical/material support: Zubkov. Study supervision: Härtl, Santos.

\section{Correspondence}

Roger Härtl, Weill Cornell Brain and Spine Center, Department of Neurological Surgery, Weill Cornell Medicine, NewYorkPresbyterian Hospital, 525 East 68th St., Box 99, New York, NY 10065. email: roger@hartlmd.net. 